\title{
Nutrient Uptake under Inter-Cropping Systems of Ginger and Cowpea and Weed Management Practices
}

\author{
A. Baruah ${ }^{1 *}$ and J. Deka ${ }^{2}$ \\ ${ }^{1}$ Dow Agro Sciences India Pvt. Ltd., Kolkata-700157, West Bengal, India \\ ${ }^{2}$ Assam Agricultural University, Jorhat-785013, Assam, India \\ *Corresponding author
}

\section{A B S T R A C T}

\begin{tabular}{|l|}
\hline K e y w o r d s \\
$\begin{array}{l}\text { Nutrient uptake, Inter- } \\
\text { cropping, Weed } \\
\text { management, Hand } \\
\text { weeding, Metribuzin, } \\
\text { Oxadiargyl, Rhizome }\end{array}$ \\
\hline Article Info \\
\hline $\begin{array}{l}\text { Accepted: } \\
\text { 22 October } 2018 \\
\text { Available Online: } \\
\text { 10 November } 2018\end{array}$ \\
\hline
\end{tabular}

A field experiment was conducted in Assam Agricultural University, Jorhat district, Assam, India to understand the effectiveness of inter-cropping system and chemical weed management on nutrient uptake by ginger (Zingiber officinale Rosc.), inter-crop and weeds, during 2013-14 and 2014-15. There were 16 numbers of treatment combinations, comprising of 4 inter-cropping systems and 4 weed management practices. Intercropping of Cowpea in between rows of Ginger and incorporated at 40 days after sowing (DAS) and treatment with Metribuzin $500 \mathrm{~g}^{\mathrm{ai}} \mathrm{ha}^{-1}+$ hand weeding $(\mathrm{HW})$ at 70, 100 and 140 days after planting (DAP) recorded better results in terms of crop yield and nutrient uptake by ginger and cowpea. On the contrary, weedy check recorded the highest nutrition uptake by weeds at almost all the considered time intervals. At the time of ginger harvest, treatments Ginger + Cowpea (2:1) and Cowpea incorporated at 40 DAS and Hand weeding (HW) at 40, 70, 100 and 140 DAP recorded better results in terms of $\mathrm{P}_{2} \mathrm{O}_{5}$ and $\mathrm{K}_{2} \mathrm{O}$ availability in soil.

\section{Introduction}

In India, Ginger (Zingiber officinale Rosc.) is considered to be a high valued commercial crop due to its multipurpose uses. Also, India is one of the major producer and consumer of ginger, accounting for about $30-40 \%$ of the global share of production, followed by China, Nepal and Indonesia (Gracy et al., 2013). But ginger is an exhaustive crop, therefore nutrient availability and uptake is critical for its production. Due to wider spacing, it faces severe competition for nutrition from weeds. Growing of intercrops and application of preemergence herbicide can be an effective approach towards better nutrient uptake by ginger, its enhanced growth and yield due to effective weed management. The current study was undertaken with the intent to understand the impact of inter-crops and chemical weed management on nutrient uptake by crops and weeds and yield of ginger.

\section{Materials and Methods}

\section{Soils characteristics}

Prior to initiating the experiment, representative soil samples were collected randomly and analysed for their chemical 
properties. The texture of the soil was found to be sandy loam with acidic in reaction. For both the years i.e. 2013-14 and 2014-15, available Nitrogen $(\mathrm{N})$ and Potassium $\left(\mathrm{K}_{2} \mathrm{O}\right)$ were in medium range whereas Phosphorus $\left(\mathrm{P}_{2} \mathrm{O}_{5}\right)$ was low. A detail description of chemical properties of the soil and methods used are presented in Table 1.

\section{Treatment details}

There were 16 treatment combinations comprising of 4 Cropping Systems viz., $\mathrm{I}_{1}$ : Ginger + Cowpea (2:1); Cowpea incorporated at 40 DAS, $\mathrm{I}_{2}$ : Ginger + Cowpea (3:1); Cowpea incorporated at $40 \mathrm{DAS}, \mathrm{I}_{3}$ : Cowpea in between rows of Ginger and incorporated at 40 DAS, $\mathrm{I}_{4}$ : Cowpea in between alternate rows of Ginger and incorporated at 40 DAS and 4 Weed Management Practices viz., $\mathrm{W}_{1}$ : Weedy (Control), $\mathrm{W}_{2}$ : Hand weeding at 40, 70, 100 and 140 DAP, $\mathrm{W}_{3}$ : Pre-emergence application of Oxadiargyl $90 \mathrm{~g}$ ai ha ${ }^{-1}+$ hand weeding at 70, 100 and 140 DAP and $\mathrm{W}_{4}$ : Pre-emergence application of Metribuzin $500 \mathrm{~g}$ ai ha $\mathrm{ha}^{-1}+$ hand weeding at 70, 100 and 140 DAP.

\section{Planting/sowing and inter-cultural operations}

The ginger variety 'Nadia' pre-treated with Mancozeb were planted in a spacing of $60 \mathrm{~cm}$ between two rows and $25 \mathrm{~cm}$ between rhizomes. The number of ginger rows was as per the row ratios in the cropping system treatments. A short duration fodder variety of cowpea 'UPC-278' was sown as an inter-crop as per the treatment requirement. The herbicides, Metribuzin and Oxadiargyl were applied with a spray volume of $5001 \mathrm{ha}^{-1}$ on the $3^{\text {rd }}$ day after planting of ginger rhizomes.

The plots were mulched with rice straw @ $4 \mathrm{t}$ $\mathrm{ha}^{-1}$ in two splits, one immediately after planting of ginger and second at 70 DAP. Light earthing up of ginger was done in all the plots at 60 and 100 DAP, except the Weedy plots. Need based plant protection measures were adopted to manage pests and diseases as per package and practices recommended by Assam Agricultural University. In both the years, cowpea was uprooted and incorporated in the soil on the $40^{\text {th }}$ day after sowing and ginger crop was harvested on the $262^{\text {nd }}$ day after planting in both the years.

\section{Results and Discussion}

\section{Effect on yield of crops}

In 2013-14 and 2014-15, treatment Cowpea in between Ginger and incorporated at 40 DAS recorded significantly higher fresh biomass weight of cowpea whereas weed management with Metribuzine $500 \mathrm{~g} \mathrm{ha}^{-1}$ pre-em+ HW 70, 100 and 140 DAP recorded significantly higher fresh biomass weight of cowpea in both the years (Table 2).

Intercropping of Cowpea in between Ginger and incorporated at 40 DAS recorded highest ginger yield of $7542 \mathrm{~kg} \mathrm{ha}^{-1}$ in 2013-14 and $8633 \mathrm{~kg} \mathrm{ha}^{-1}$ in 2014-15. It was statistically at par with the treatment Cowpea in alternate rows and incorporated at 40 DAS. Application of Metribuzine $500 \mathrm{~g} \mathrm{ha}^{-1}$ pre-em+ HW 70, 100 and 140 DAP recorded significantly higher ginger yield of 7817and $9340 \mathrm{~kg} \mathrm{ha}^{-1}$ in 2013-14 and 2014-15, respectively as compared to other weed management treatment (Table 2).

\section{Effect on nutrient uptake by crops}

In both the years of experimentation, highest Nitrogen (N) uptake of $98.8 \mathrm{~kg} \mathrm{ha}^{-1}(2013-14)$ and $114.7 \mathrm{~kg} \mathrm{ha}^{-1}(2014-15)$ by ginger was recorded in the treatment Cowpea in between Ginger and incorporated at 40 DAS. It was statistically at par with the treatment Cowpea in alternate rows and incorporated at 40 DAS. Highest Phosphorus (P) uptake by ginger was 
also recorded in the treatment Cowpea in between Ginger and incorporated at 40 DAS, which recorded uptake of $11.5 \mathrm{~kg} \mathrm{ha}^{-1}$ in 2013-14 and $13.3 \mathrm{~kg} \mathrm{ha}^{-1}$ in 2014-15, but it was statistically at par with the treatment Cowpea in alternate rows and incorporated at 40 DAS. A similar trend was also observed in respect of Potassium (K) uptake by ginger.

Inter-cropping system Cowpea in between Ginger and incorporated at 40 DAS recorded the highest $\mathrm{K}$ uptake of 108.5 and $118.4 \mathrm{~kg}^{-} \mathrm{ha}^{-}$ ${ }^{1}$ in 2013-14 and 2014-15, respectively which was at par with the treatment Cowpea in alternate rows and incorporated at 40 DAS (Table 3). Thus, in both the years, intercropping treatment Cowpea in between Ginger and incorporated at 40 DAP recorded highest nutrient uptake by ginger. Better biomass production by ginger under this intercropping system due to better weed smothering at the critical stage of competition and $\mathrm{N}$ fixation by legume cowpea and its incorporation might have increased the nutrient uptake (Willey, 1979).

Amongst the weed management practices, Metribuzine $500 \mathrm{~g} \mathrm{ha}^{-1}$ pre-em + HW 70, 100 and 140 DAP recorded significantly higher $\mathrm{N}$ uptake of $106.8 \mathrm{~kg} \mathrm{ha}^{-1}$ in 2013-14 and 128.1 $\mathrm{kg} \mathrm{ha}^{-1}$ in 2014-15 by ginger. This treatment also recorded significantly higher $\mathrm{P}$ uptake of 12.4 and $14.9 \mathrm{~kg} \mathrm{ha}^{-1}$ in 2013-14 and 2014-15, respectively as compared to other weed management treatments. $\mathrm{K}$ uptake of 113.2 and $121.7 \mathrm{~kg} \mathrm{ha}^{-1}$ in 2013-14 and 2014-15, respectively by ginger was also recorded in Metribuzine $500 \mathrm{~g} \mathrm{ha}^{-1}$ pre-em + HW 70, 100 and 140 DAP (Table 3), which was significantly higher than other weed management. Nutrient uptake in Metribuzin treated plots were followed by the treatment Oxadiargyl $90 \mathrm{~g} \mathrm{ha}^{-1}$ pre-em + HW 70, 100 and 140 DAP. Metribuzin application followed by 3 hand weeding created a sustained competition free environment for better growth of ginger finally contributing towards increased nutrient uptake by the crop. Similar, results are reported by Sathiyavani and Prabhakaran (2015) also in turmeric.

Intercropping of Cowpea in between Ginger and incorporated at 40 DAS, recorded significantly higher uptake of N, P and K by cowpea as compared to all other cropping systems (Table 4). This might be due to the closer row spacing which created an ideal weed free condition. It contributed towards better biomass production leading to higher nutrient uptake by cowpea.

Weed management with Metribuzine $500 \mathrm{~g}$ ha 1 pre-em + HW 70, 100 and 140 DAP recorded significantly higher uptake of $\mathrm{N}, \mathrm{P}$ and $\mathrm{K}$ by cowpea than rest of the weed management treatments (Table 4). It was followed by the treatment Oxadiargyl $90 \mathrm{~g}$ $\mathrm{ha}^{-1}$ pre-em + HW 70, 100 and 140 DAP. Preemergence application of those two herbicides resulted effective weed control during the critical phases, thus helping better nutrient uptake by the inter-crop cowpea.

Table.1 Chemical properties of surface soil at experimental site

\begin{tabular}{|c|c|c|c|}
\hline \multirow{2}{*}{$\begin{array}{c}\text { Particular(s) } \\
\left(\mathrm{kg} \mathrm{ha}^{-1}\right)\end{array}$} & \multicolumn{2}{|c|}{ Value(s) } & \multicolumn{2}{c|}{ Method adopted } \\
\hline A vailable $\mathbf{N}$ & 388.0 & 369.40 & Kjeldahl Method (Jackson, 1973) \\
\hline A vailable $\mathrm{P}_{2} \mathrm{O}_{5}$ & 17.8 & 13.8 & Bray-I Method (Jackson, 1973) \\
\hline A vailable $\mathrm{K}_{2} \mathrm{O}$ & 239.4 & 252.3 & Flame Photometric Method (Jacson,1973) \\
\hline
\end{tabular}


Table.2 Fresh biomass weight of cowpea and rhizome yield of ginger $\left(\mathrm{kg} \mathrm{ha}^{-1}\right)$

\begin{tabular}{|c|c|c|c|c|}
\hline \multirow[t]{2}{*}{ Treatment } & \multicolumn{2}{|c|}{$\begin{array}{c}\text { Cowpea biomass weight } \\
\qquad\left(\mathrm{kg} \mathrm{ha}^{-1}\right)\end{array}$} & \multicolumn{2}{|c|}{$\begin{array}{l}\text { Ginger rhizome yield } \\
\left(\mathrm{kg} \mathrm{ha}^{-1}\right)\end{array}$} \\
\hline & 2013-14 & 2014-15 & 2013-14 & 2014-15 \\
\hline \multicolumn{5}{|l|}{ Cropping system } \\
\hline$I_{1:} G^{*}+C^{*}(2: 1) ; C$ incorp. 40 DAS & 4679 & 4523 & 5846 & 6175 \\
\hline $\mathrm{I}_{2:} \mathrm{G}+\mathrm{C}(3: 1) ; \mathrm{C}$ incorp. $40 \mathrm{DAS}$ & 3656 & 3729 & 5925 & 6454 \\
\hline $\mathrm{I}_{3:} \mathrm{C}$ in between G; incorp. 40 DAS & 14333 & 10158 & 7542 & 8633 \\
\hline $\mathrm{I}_{4:} \mathrm{C}$ in alternate rows; incorp. $40 \mathrm{DAS}$ & 9229 & 7458 & 7338 & 8505 \\
\hline $\mathrm{CD}_{\mathrm{P}=0.05}$ & 448 & 305 & 419 & 635 \\
\hline \multicolumn{5}{|l|}{ Weed management } \\
\hline$\overline{W_{1:} \text { Weedy }}$ & 7796 & 6196 & 5021 & 4825 \\
\hline $\mathrm{W}_{2:} \mathrm{HW}$ 40, 70, 100 and $140 \mathrm{DAP}$ & 7563 & 6108 & 6533 & 7396 \\
\hline $\mathrm{W}_{3}$ : Oxadiargyl $90 \mathrm{~g} \mathrm{ha}^{-1}$ pre-em+ HW 70, 100 and 140 DAP & 8043 & 6606 & 7279 & 8208 \\
\hline $\begin{array}{l}\text { W: Metribuzine } 500 \mathrm{~g} \mathrm{ha}^{-1} \text { pre-em+ HW } 70,100 \text { and } 140 \\
\text { DAP }\end{array}$ & 8497 & 6958 & 7817 & 9340 \\
\hline $\mathrm{CD}_{\mathrm{P}=0.05}$ & 448 & 305 & 338 & 635 \\
\hline
\end{tabular}


Table.3 Nutrient (N, P and K) uptake by ginger $\left(\mathrm{kg} \mathrm{ha}^{-1}\right)$

\begin{tabular}{|c|c|c|c|c|c|c|}
\hline \multirow{2}{*}{ Treatments } & \multicolumn{2}{|c|}{$\mathbf{N}\left(\mathrm{kg} \mathrm{ha}^{-1}\right)$} & \multicolumn{2}{|c|}{$\mathbf{P}\left(\mathrm{kg} \mathrm{ha}^{-1}\right)$} & \multicolumn{2}{|c|}{$\mathrm{K}\left(\mathrm{kg} \mathrm{ha}^{-1}\right)$} \\
\hline & $\begin{array}{l}2013- \\
14\end{array}$ & 2014-15 & $\begin{array}{l}2013- \\
14\end{array}$ & 2014-15 & $\begin{array}{l}2013- \\
14\end{array}$ & $\begin{array}{l}2014- \\
15\end{array}$ \\
\hline \multicolumn{7}{|l|}{ Cropping system } \\
\hline$I_{1:} G^{*}+C^{*}(2: 1) ; C$ incorp. 40 DAS & 66.7 & 71.1 & 8.2 & 8.7 & 72.2 & 76.7 \\
\hline$I_{2:} G+C(3: 1) ; C$ incorp. 40 DAS & 67.6 & 74.1 & 8.3 & 9.1 & 73.2 & 80.0 \\
\hline $\mathrm{I}_{3:} \mathrm{C}$ in between G; incorp. 40 DAS & 98.8 & 114.7 & 11.5 & 13.3 & 108.5 & 118.4 \\
\hline $\mathrm{I}_{4:} \mathrm{C}$ in alternate rows; incorp. $40 \mathrm{DAS}$ & 96.1 & 113.0 & 11.2 & 13.1 & 105.6 & 116.5 \\
\hline $\mathrm{CD}_{\mathrm{P}=0.05}$ & 4.3 & 2.4 & 0.5 & 0.3 & 4.6 & 4.5 \\
\hline \multicolumn{7}{|l|}{ Weed management } \\
\hline W $_{1:}$ Weedy & 40.1 & 38.5 & 5.5 & 5.3 & 52.7 & 50.7 \\
\hline $\mathrm{W}_{2:} \mathrm{HW}$ 40, 70, 100 and 140 DAP & 83.2 & 94.4 & 9.8 & 11.1 & 88.7 & 100.7 \\
\hline $\mathrm{W}_{3}$ : Oxadiargyl $90 \mathrm{~g} \mathrm{ha}^{-1}$ pre-em+ HW 70, 100 and 140 DAP & 99.1 & 111.9 & 11.5 & 13.0 & 105.0 & 118.5 \\
\hline $\mathrm{W}_{4:}$ Metribuzine $500 \mathrm{~g} \mathrm{ha}^{-1}$ pre-em+ HW 70, 100 and 140 DAP & 106.8 & 128.1 & 12.4 & 14.9 & 113.2 & 121.7 \\
\hline$C D_{P=0.05}$ & 4.3 & 4.1 & 0.5 & 0.3 & 4.6 & 4.5 \\
\hline
\end{tabular}


Table.4 Nutrient (N, P and K) uptake by cowpea $\left(\mathrm{kg} \mathrm{ha}^{-1}\right)$

\begin{tabular}{|c|c|c|c|c|c|c|}
\hline \multirow[t]{2}{*}{ Treatments } & \multicolumn{2}{|c|}{$\mathbf{N}\left(\mathbf{k g ~ h a}^{-1}\right)$} & \multicolumn{2}{|c|}{$\mathbf{P}\left(\mathrm{kg} \mathrm{ha}^{-1}\right)$} & \multicolumn{2}{|c|}{ K $\left(\mathrm{kg} \mathrm{ha}^{-1}\right)$} \\
\hline & $\begin{array}{c}2013- \\
14\end{array}$ & 2014-15 & $\begin{array}{c}2013- \\
14\end{array}$ & 2014-15 & $\begin{array}{c}2013- \\
14\end{array}$ & $\begin{array}{c}2014- \\
15\end{array}$ \\
\hline \multicolumn{7}{|l|}{ Cropping system } \\
\hline$I_{1:} G^{*}+C *(2: 1) ; C$ incorp. 40 DAS & 44.0 & 41.5 & 6.7 & 6.4 & 54.0 & 52.3 \\
\hline $\mathrm{I}_{2:} \mathbf{G}+\mathrm{C}(3: 1) ; \mathrm{C}$ incorp. 40 DAS & 34.3 & 35.1 & 5.2 & 5.3 & 42.0 & 43.0 \\
\hline $\mathrm{I}_{3:} \mathrm{C}$ in between G; incorp. $40 \mathrm{DAS}$ & 160.3 & 111.2 & 21.9 & 15.6 & 195.0 & 137.2 \\
\hline $\mathrm{I}_{4:} \mathrm{C}$ in alternate rows; incorp. $40 \mathrm{DAS}$ & 106.7 & 82.8 & 14.1 & 11.2 & 127.1 & 100.2 \\
\hline $\mathrm{CD}_{\mathrm{P}=0.05}$ & 4.4 & 2.6 & 0.4 & 0.5 & 3.9 & 3.6 \\
\hline \multicolumn{7}{|l|}{ Weed management } \\
\hline $\mathrm{W}_{1:}$ Weedy & 79.8 & 63.0 & 11.5 & 9.1 & 94.4 & 74.5 \\
\hline$W_{2:} H W 40,70,100$ and 140 DAP & 79.0 & 62.1 & 11.1 & 9.0 & 91.6 & 73.5 \\
\hline $\mathrm{W}_{3}$ : Oxadiargyl $90 \mathrm{~g} \mathrm{ha}^{-1}$ pre-em+ HW 70, 100 and 140 DAP & 90.1 & 70.7 & 12.3 & 10.1 & 114.8 & 91.1 \\
\hline $\mathrm{W}_{4:}$ Metribuzine $500 \mathrm{~g} \mathrm{ha}^{-1}$ pre-em+ HW 70, 100 and 140 DAP & 94.7 & 74.7 & 12.9 & 10.7 & 119.4 & 94.8 \\
\hline $\mathrm{CD}_{\mathrm{P}=0.05}$ & 4.4 & 2.6 & 0.4 & 0.5 & 3.9 & 3.6 \\
\hline
\end{tabular}




\section{Int.J.Curr.Microbiol.App.Sci (2018) 7(11): 2858-2870}

Table.5 Nitrogen $\left(\mathrm{kg} \mathrm{ha}^{-1}\right)$ uptake by weeds

\begin{tabular}{|c|c|c|c|c|c|c|c|c|c|c|}
\hline \multirow{2}{*}{ Treatments } & \multicolumn{5}{|c|}{ 2013-14 } & \multicolumn{5}{|c|}{ 2014-15 } \\
\hline & $\begin{array}{c}40 \\
\text { DAP }\end{array}$ & $\begin{array}{c}70 \\
\text { DAP }\end{array}$ & $\begin{array}{c}100 \\
\text { DAP }\end{array}$ & $\begin{array}{c}130 \\
\text { DAP }\end{array}$ & $\begin{array}{c}160 \\
\text { DAP }\end{array}$ & $\begin{array}{c}40 \\
\text { DAP }\end{array}$ & $\begin{array}{c}70 \\
\text { DAP }\end{array}$ & $\begin{array}{c}100 \\
\text { DAP }\end{array}$ & $\begin{array}{c}130 \\
\text { DAP }\end{array}$ & $\begin{array}{c}160 \\
\text { DAP }\end{array}$ \\
\hline \multicolumn{11}{|l|}{ Cropping system } \\
\hline$I_{1:} G^{*}+C^{*}(2: 1) ; C$ incorp. 40 DAS & 23.2 & 55.2 & 72.5 & 84.5 & 87.4 & 20.7 & 49.7 & 66.9 & 78.7 & 82.9 \\
\hline $\mathrm{I}_{2:} \mathbf{G}+\mathrm{C}(3: 1) ; \mathrm{C}$ incorp. 40 DAS & 23.3 & 47.0 & 62.0 & 71.7 & 75.8 & 20.9 & 42.3 & 57.2 & 66.8 & 70.8 \\
\hline $\mathrm{I}_{3:} \mathrm{C}$ in between G; incorp. 40 DAS & 13.5 & 28.4 & 38.6 & 47.4 & 50.3 & 12.1 & 24.9 & 37.1 & 43.6 & 46.1 \\
\hline $\mathrm{I}_{4:} \mathrm{C}$ in alternate rows; incorp. $40 \mathrm{DAS}$ & 15.0 & 31.6 & 41.8 & 49.5 & 52.3 & 13.6 & 28.1 & 36.1 & 45.6 & 48.4 \\
\hline $\mathrm{CD}_{\mathrm{P}=0.05}$ & 2.0 & 1.4 & 2.6 & 2.2 & 2.7 & 1.0 & 1.4 & 1.8 & 2.9 & 3.4 \\
\hline \multicolumn{11}{|l|}{ Weed management } \\
\hline $\mathrm{W}_{1:}$ Weedy & 35.1 & 103.6 & 131.3 & 150.5 & 155.0 & 29.1 & 96.3 & 123.6 & 142.6 & 148.5 \\
\hline $\mathrm{W}_{2:} \mathrm{HW} 40,70,100$ and 140 DAP & 20.8 & 21.1 & 30.1 & 37.0 & 39.5 & 30.0 & 17.9 & 26.8 & 33.6 & 36.2 \\
\hline $\mathrm{W}_{3}$ : Oxadiargyl $90 \mathrm{~g} \mathrm{ha}^{-1}$ pre-em+ HW 70, 100 and 140 DAP & 11.0 & 19.4 & 27.5 & 33.7 & 36.5 & 9.9 & 16.1 & 24.2 & 30.5 & 33.2 \\
\hline $\mathrm{W}_{4:}$ Metribuzine $500 \mathrm{~g} \mathrm{ha}^{-1}$ pre-em+ HW 70, 100 and 140 DAP & 8.0 & 18.1 & 26.0 & 31.8 & 34.7 & 6.9 & 14.8 & 22.8 & 27.9 & 30.4 \\
\hline $\mathrm{CD}_{\mathrm{P}=0.05}$ & 2.0 & 1.4 & 2.6 & 2.2 & 2.7 & 1.0 & 1.4 & 1.8 & 2.9 & 3.4 \\
\hline
\end{tabular}




\section{Int.J.Curr.Microbiol.App.Sci (2018) 7(11): 2858-2870}

Table.6 Phosphorus $\left(\mathrm{kg} \mathrm{ha}^{-1}\right)$ uptake by weeds

\begin{tabular}{|c|c|c|c|c|c|c|c|c|c|c|}
\hline \multirow[t]{2}{*}{ Treatments } & \multicolumn{5}{|c|}{ 2013-14 } & \multicolumn{5}{|c|}{ 2014-15 } \\
\hline & $\begin{array}{c}40 \\
\text { DAP }\end{array}$ & $\begin{array}{c}70 \\
\text { DAP }\end{array}$ & $\begin{array}{c}100 \\
\text { DAP }\end{array}$ & $\begin{array}{c}130 \\
\text { DAP }\end{array}$ & $\begin{array}{c}160 \\
\text { DAP }\end{array}$ & $\begin{array}{c}40 \\
\text { DAP }\end{array}$ & $\begin{array}{c}70 \\
\text { DAP }\end{array}$ & $\begin{array}{c}100 \\
\text { DAP }\end{array}$ & $\begin{array}{c}130 \\
\text { DAP }\end{array}$ & $\begin{array}{c}160 \\
\text { DAP }\end{array}$ \\
\hline \multicolumn{11}{|l|}{ Cropping system } \\
\hline$I_{1:} G^{*}+C^{*}(2: 1) ; C$ incorp. 40 DAS & 3.27 & 7.4 & 9.8 & 11.5 & 12.1 & 3.0 & 6.6 & 9.0 & 10.7 & 11.3 \\
\hline $\mathrm{I}_{2:} \mathbf{G}+\mathrm{C}(3: 1) ; \mathrm{C}$ incorp. $40 \mathrm{DAS}$ & 3.50 & 6.4 & 8.5 & 9.8 & 10.4 & 3.0 & 5.7 & 7.7 & 9.1 & 9.7 \\
\hline $\mathrm{I}_{3:} \mathrm{C}$ in between G; incorp. 40 DAS & 2.00 & 3.8 & 5.2 & 6.4 & 6.8 & 1.8 & 3.3 & 4.8 & 5.7 & 6.2 \\
\hline $\mathrm{I}_{4:} \mathrm{C}$ in alternate rows; incorp. 40 DAS & 2.16 & 4.1 & 5.6 & 6.7 & 7.0 & 2.0 & 3.7 & 4.8 & 6.1 & 6.5 \\
\hline $\mathrm{CD}_{\mathrm{P}=0.05}$ & 0.28 & 0.2 & 0.5 & 0.4 & 0.5 & 0.2 & 0.2 & 0.4 & 0.4 & 0.5 \\
\hline \multicolumn{11}{|l|}{ Weed management } \\
\hline$W_{1:}$ Weedy & 4.13 & 12.2 & 15.4 & 17.7 & 18.4 & 3.5 & 11.3 & 14.4 & 16.8 & 17.4 \\
\hline $\mathrm{W}_{2:} \mathrm{HW} 40,70,100$ and $140 \mathrm{DAP}$ & 3.53 & 3.6 & 5.1 & 6.2 & 6.8 & 3.7 & 3.0 & 4.5 & 5.6 & 6.1 \\
\hline $\mathrm{W}_{3}$ : Oxadiargyl $90 \mathrm{~g} \mathrm{ha}^{-1}$ pre-em+ HW 70, 100 and $140 \mathrm{DAP}$ & 1.76 & 3.1 & 4.4 & 5.4 & 5.8 & 1.5 & 2.5 & 3.9 & 4.8 & 5.3 \\
\hline $\mathrm{W}_{4:}$ Metribuzine $500 \mathrm{~g} \mathrm{ha}^{-1}$ pre-em+ HW 70, 100 and $140 \mathrm{DAP}$ & 1.51 & 2.9 & 4.0 & 5.1 & 5.4 & 1.4 & 2.4 & 3.5 & 4.5 & 4.9 \\
\hline $\mathrm{CD}_{\mathrm{P}=0.05}$ & 0.28 & 0.2 & 0.5 & 0.4 & 0.5 & 0.2 & 0.2 & 0.4 & 0.4 & 0.5 \\
\hline
\end{tabular}

$\mathrm{G}^{*}=$ Ginger,$\quad \mathrm{C}^{*}$ Cowpea, $\quad$ pre-em=Pre-emergence 
Table.7 Potassium $\left(\mathrm{kg} \mathrm{ha}^{-1}\right)$ uptake by weeds

\begin{tabular}{|c|c|c|c|c|c|c|c|c|c|c|}
\hline \multirow[t]{2}{*}{ Treatments } & \multicolumn{5}{|c|}{ 2013-14 } & \multicolumn{5}{|c|}{ 2014-15 } \\
\hline & $\begin{array}{c}40 \\
\text { DAP }\end{array}$ & $\begin{array}{c}70 \\
\text { DAP }\end{array}$ & $\begin{array}{c}100 \\
\text { DAP }\end{array}$ & $\begin{array}{c}130 \\
\text { DAP }\end{array}$ & $\begin{array}{c}160 \\
\text { DAP }\end{array}$ & $\begin{array}{c}40 \\
\text { DAP }\end{array}$ & $\begin{array}{c}70 \\
\text { DAP }\end{array}$ & $\begin{array}{c}100 \\
\text { DAP }\end{array}$ & $\begin{array}{c}130 \\
\text { DAP }\end{array}$ & $\begin{array}{c}160 \\
\text { DAP }\end{array}$ \\
\hline \multicolumn{11}{|l|}{ Cropping system } \\
\hline$I_{1}: G^{*}+C^{*}(2: 1) ; C$ incorp. 40 DAS & 28.8 & 66.9 & 87.6 & 103.2 & 108.6 & 25.8 & 60.3 & 81.4 & 96.2 & 101.6 \\
\hline$I_{2:} G+C(3: 1) ; C$ incorp. 40 DAS & 29.4 & 55.4 & 73.6 & 85.4 & 89.6 & 26.2 & 49.8 & 67.4 & 79.3 & 84.4 \\
\hline $\mathrm{I}_{3:} \mathrm{C}$ in between G; incorp. 40 DAS & 17.4 & 34.7 & 46.6 & 58.5 & 61.2 & 15.6 & 30.5 & 45.3 & 53.7 & 56.1 \\
\hline $\mathrm{I}_{4:} \mathrm{C}$ in alternate rows; incorp. 40 DAS & 19.4 & 37.3 & 53.4 & 61.0 & 63.7 & 17.6 & 32.7 & 43.8 & 55.4 & 59.1 \\
\hline $\mathrm{CD}_{\mathrm{P}=0.05}$ & 2.5 & 1.6 & 3.4 & 2.8 & 2.9 & 1.2 & 1.5 & 1.8 & 3.0 & 4.3 \\
\hline \multicolumn{11}{|l|}{ Weed management } \\
\hline $\mathrm{W}_{1:}$ Weedy & 37.4 & 110.1 & 139.7 & 159.9 & 164.6 & 28.9 & 102.4 & 131.4 & 151.6 & 157.8 \\
\hline $\mathrm{W}_{2:} \mathrm{HW} 40,70,100$ and $140 \mathrm{DAP}$ & 30.7 & 31.0 & 44.3 & 54.4 & 58.1 & 30.1 & 26.1 & 39.3 & 49.4 & 53.2 \\
\hline $\mathrm{W}_{3}$ : Oxadiargyl $90 \mathrm{~g} \mathrm{ha}^{-1}$ pre-em+ HW 70, 100 and 140 DAP & 15.5 & 27.4 & 39.8 & 48.0 & 51.5 & 14.0 & 23.1 & 34.3 & 43.3 & 47.2 \\
\hline $\mathrm{W}_{4:}$ Metribuzine $500 \mathrm{~g} \mathrm{ha}^{-1}$ pre-em+ HW 70, 100 and $140 \mathrm{DAP}$ & 11.3 & 25.8 & 37.4 & 45.9 & 48.9 & 9.7 & 21.7 & 32.9 & 40.4 & 43.1 \\
\hline $\mathrm{CD}_{\mathrm{P}=0.05}$ & 2.5 & 1.6 & 3.4 & 2.8 & 2.9 & 1.2 & 1.5 & 1.8 & 3.0 & 4.3 \\
\hline
\end{tabular}


Table.8 Available nutrients $\left(\mathrm{kg} \mathrm{ha}^{-1}\right)$ in the soil at the time of cowpea incorporation

\begin{tabular}{|c|c|c|c|c|c|c|}
\hline \multirow[t]{2}{*}{ Treatments } & \multicolumn{2}{|c|}{$\mathbf{N}\left(\mathrm{kg} \mathrm{ha}^{-1}\right)$} & \multicolumn{2}{|c|}{$\mathbf{P}\left(\mathrm{kg} \mathrm{ha}^{-1}\right)$} & \multicolumn{2}{|c|}{$\mathrm{K}\left(\mathrm{kg} \mathrm{ha}^{-1}\right)$} \\
\hline & $\begin{array}{c}2013- \\
14\end{array}$ & 2014-15 & $\begin{array}{c}2013- \\
14\end{array}$ & 2014-15 & $\begin{array}{c}2013- \\
14\end{array}$ & $\begin{array}{c}2014- \\
15\end{array}$ \\
\hline \multicolumn{7}{|l|}{ Cropping system } \\
\hline$I_{1:} G^{*}+C *(2: 1) ; C$ incorp. 40 DAS & 264.2 & 266.3 & 35.9 & 37.2 & 83.9 & 91.3 \\
\hline $\mathrm{I}_{2:} \mathrm{G}+\mathrm{C}(3: 1) ; \mathrm{C}$ incorp. 40 DAS & 255.5 & 270.4 & 29.3 & 30.7 & 76.2 & 82.0 \\
\hline $\mathrm{I}_{3:} \mathrm{C}$ in between G; incorp. 40 DAS & 280.0 & 282.2 & 20.7 & 22.0 & 54.1 & 65.3 \\
\hline $\mathrm{I}_{4} \mathrm{C}$ in alternate rows; incorp. $40 \mathrm{DAS}$ & 272.6 & 276.2 & 23.3 & 24.6 & 64.3 & 74.3 \\
\hline $\mathrm{CD}_{\mathrm{P}=0.05}$ & NS & NS & 3.6 & 3.7 & 5.4 & 3.9 \\
\hline \multicolumn{7}{|l|}{ Weed management } \\
\hline $\mathrm{W}_{1:}$ Weedy & 265.0 & 267.8 & 26.0 & 27.4 & 67.6 & 76.0 \\
\hline $\mathrm{W}_{2:} \mathrm{HW}$ 40, 70, 100 and 140 DAP & 270.8 & 278.7 & 28.7 & 30.0 & 72.4 & 80.8 \\
\hline $\mathrm{W}_{3}$ : Oxadiargyl $90 \mathrm{~g} \mathrm{ha}^{-1}$ pre-em+ HW 70, 100 and 140 DAP & 268.5 & 276.2 & 27.8 & 29.1 & 69.7 & 78.6 \\
\hline $\mathrm{W}_{4:}$ Metribuzine $500 \mathrm{~g} \mathrm{ha}^{-1}$ pre-em+ HW 70, 100 and 140 DAP & 267.9 & 272.5 & 26.7 & 28.0 & 68.7 & 77.5 \\
\hline $\mathrm{CD}_{\mathrm{P}=0.05}$ & NS & NS & NS & NS & NS & NS \\
\hline
\end{tabular}




\section{Int.J.Curr.Microbiol.App.Sci (2018) 7(11): 2858-2870}

Table.9 Available nutrients $\left(\mathrm{kg} \mathrm{ha}^{-1}\right)$ in the soil at the time of ginger incorporation

\begin{tabular}{|c|c|c|c|c|c|c|}
\hline \multirow[t]{2}{*}{ Treatments } & \multicolumn{2}{|c|}{$\mathbf{N}\left(\mathrm{kg} \mathrm{ha}^{-1}\right)$} & \multicolumn{2}{|c|}{$\mathbf{P}\left(\mathrm{kg} \mathrm{ha}^{-1}\right)$} & \multicolumn{2}{|c|}{$\mathrm{K}\left(\mathrm{kg} \mathrm{ha}^{-1}\right)$} \\
\hline & $\begin{array}{c}2013- \\
14\end{array}$ & 2014-15 & $\begin{array}{c}2013- \\
14\end{array}$ & 2014-15 & $\begin{array}{c}2013- \\
14\end{array}$ & $\begin{array}{c}\text { 2014- } \\
15\end{array}$ \\
\hline \multicolumn{7}{|l|}{ Cropping system } \\
\hline $\mathrm{I}_{1:} \mathrm{G}^{*}+\mathrm{C}^{*}(2: 1) ; \mathrm{C}$ incorp. $40 \mathrm{DAS}$ & 269.1 & 260.3 & 30.5 & 31.7 & 73.2 & 74.5 \\
\hline $\mathrm{I}_{2:} \mathrm{G}+\mathrm{C}(3: 1) ; \mathrm{C}$ incorp. 40 DAS & 264.8 & 257.1 & 26.3 & 27.6 & 67.0 & 68.4 \\
\hline I3: C in between G; incorp. 40 DAS & 279.9 & 274.3 & 19.6 & 20.6 & 50.8 & 52.2 \\
\hline $\mathrm{I}_{4:} \mathrm{C}$ in alternate rows; incorp. $40 \mathrm{DAS}$ & 277.9 & 260.3 & 21.0 & 22.9 & 59.0 & 60.3 \\
\hline $\mathrm{CD}_{\mathrm{P}=0.05}$ & NS & NS & 3.2 & 2.7 & 4.7 & 4.7 \\
\hline \multicolumn{7}{|l|}{ Weed management } \\
\hline$\overline{\mathrm{W}_{1} \text { : Weedy }}$ & 266.5 & 255.4 & 17.9 & 19.1 & 47.3 & 48.6 \\
\hline $\mathrm{W}_{2:} \mathrm{HW}$ 40, 70, 100 and $140 \mathrm{DAP}$ & 278.0 & 266.1 & 27.3 & 28.7 & 69.9 & 71.2 \\
\hline $\mathrm{W}_{3}$ : Oxadiargyl $90 \mathrm{~g} \mathrm{ha}^{-1}$ pre-em+ HW 70, 100 and 140 DAP & 274.5 & 265.4 & 26.5 & 27.9 & 67.1 & 68.4 \\
\hline $\mathrm{W}_{4:}$ Metribuzine $500 \mathrm{~g} \mathrm{ha}^{-1}$ pre-em+ HW 70, 100 and 140 DAP & 272.7 & 265.2 & 25.7 & 27.2 & 65.8 & 67.1 \\
\hline $\mathrm{CD}_{\mathrm{P}=0.05}$ & NS & NS & 3.2 & 2.7 & 4.7 & 4.7 \\
\hline
\end{tabular}




\section{Effect on nutrient uptake by weeds}

In both the years of experimentation, highest $\mathrm{N}$ uptake by weeds during initial phase (40 DAP) was recorded in the treatment Ginger + Cowpea (3:1) and Cowpea incorporated at 40 DAS, which was at par with Ginger + Cowpea (2:1) and Cowpea incorporated at 40 DAS. But at subsequent stages (70, 100, 130 and 160 DAP), significantly higher $\mathrm{N}$ uptake was recorded in the treatment Ginger + Cowpea (2:1) and Cowpea incorporated at 40 DAS. On the other hand, the lowest $\mathrm{N}$ uptake was by weeds was recorded under Cowpea in between Ginger and incorporated at 40 DAS at all the stages in both the years (Table 5). Highest $\mathrm{P}$ and $\mathrm{K}$ uptake was recorded at 70 , 100, 130 and 160 DAP, respectively in the treatment Ginger + Cowpea (2:1) and Cowpea incorporated at 40 DAS. The treatment Cowpea in between Ginger and incorporated at 40 DAS recorded the lowest $\mathrm{P}$ and $\mathrm{K}$ uptake by weeds at all the stages of observation in both the years (Table 6 and 7). Increasing intercrop density showed better suppression of weeds resulting lower nutrient uptake by weed.

Application of Metribuzine $500 \mathrm{~g} \mathrm{ha}^{-1}$ preem+ HW 70, 100 and 140 DAP at all the stages in both the years showed the lowest uptake of $\mathrm{N}, \mathrm{P}$ and $\mathrm{K}$ by weeds as compared to the other weed management treatments (Table 5, 6 and 7). The result trend of weed density and weed dry weight resembled the trend of uptake of different nutrients at various stages of growth. This result is in concomitance with the reports of Sathiyavani (2014) in turmeric.

\section{Effect on available nutrients in soil}

Intercropping systems had no significant effect on the available $\mathrm{N}$ in the soil at the time of cowpea incorporation in both the years. However, Ginger + Cowpea (2:1) and
Cowpea incorporated at 40 DAS resulted significantly higher amount of $\mathrm{P}_{2} \mathrm{O}_{5}$ and $\mathrm{K}_{2} \mathrm{O}$ at the time of cowpea incorporation in both the years. Different weed management practices had no effect on the available nutrients in the soil at the time of cowpea incorporation in both the years (Table 8).

Available $\mathrm{N}$ in the soil at the time of ginger harvest in both the years of experimentation was not impacted by the intercropping systems. But significantly higher available $\mathrm{P}_{2} \mathrm{O}_{5}$ and $\mathrm{K}_{2} \mathrm{O}$ was recorded in Ginger + Cowpea (2:1) and Cowpea incorporated at 40 DAS in both the years in relation to other cropping systems. Different weed management practices had no significant effect on the available $\mathrm{N}$ in the soil at the time of ginger harvest in both the years. Hand weeding at 40, 70, 100 and 140 DAP recorded highest available $\mathrm{P}_{2} \mathrm{O}_{5}$ in both the years. Similarly, this treatment also recorded highest available $\mathrm{K}_{2} \mathrm{O}$ at the time of ginger harvest (Table 9). It might be due to lesser uptake of nutrients by the crop as compared to integrated weed management treatments using pre-emergence herbicides.

\section{References}

Gracy, C. P.; Jyoti, N. J. and Nagashree, N. (2013). Ginger price forecast - Store and sell ginger. Agropedia.

Palaniappan, S. P. (1988). Cropping system in the tropics. In: Principles and Management. Wiley Eastern Limited, New Delhi.

Sathiyavani, E. (2014). Evaluation of integrated weed management with pre and post emergence herbicides in turmeric. Ph.D. Thesis. Directorate of crop management, Tamil Nadu Agricultural University, Coimbatore.

Sathiyavani, E. and Prabhakaran, N. K. (2015). Effect of integrated weed management practices on plant height, 
number of tillers in turmeric during kharif season. International Journal of Horticulture 5(2): 1-8.

Willey, R. W. (1979). A scientific approach to intercropping research. In: The
Proceeding of the International Workshop on Intercropping. ICRISAT, Hyderabad, 10-13 Jan.

\section{How to cite this article:}

Baruah, A. and Deka, J. 2018. Nutrient Uptake under Inter-Cropping Systems of Ginger and Cowpea and Weed Management Practices. Int.J.Curr.Microbiol.App.Sci. 7(11): 2858-2870. doi: https://doi.org/10.20546/ijcmas.2018.711.329 